\title{
HELIOSEISMIC CONSTRAINTS ON THE STRUCTURE OF THE PRESENT SUN
}

\author{
V.A.BATURIN AND S.V.AJUKOV \\ Sternberg Astronomical Institute, Moscow 119899, Russia
}

\section{INTRODUCTION}

Given the chemical composition (essentially the hydrogen abundance), mass and the physics of the interior, the structure of a star is uniquely determined. The usual method for the determination of the hydrogen-abundance profile of the present Sun is via evolutionary calculations. But the existing uncertainties in the physics and evolutionary history suggest considering a more extended set of possible solar models. Here, we examine models with solar values of radius, luminosity and mass, but with arbitrary profiles of the hydrogen abundance in the core. Of course, the profiles $X(m / M)$ must satisfy some (rather weak) restrictions, so that the solar values (determined by fitting) can be attained.

We assume the hypothesis that within fixed physics (opacity, thermodynamics, nuclear reactions) there is for all solar models a rigorous relation between the surface helium abundance and the entropy of the adiabatic part of the convective zone. We verify this hypothesis with models using somewhat simplified physics (Christensen-Dalsgaard 1988), and with six evolutionary models computed by Christensen-Dalsgaard (1992). The relation mainly depends on the opacity in the radiative zone between the lower boundary of convection and the region of nuclear reactions. We discuss the possibility of an agreement between these relations and the helioseismic determination of the surface helium abundance.

\section{SOLAR MODELS WITH FIXED ENVELOPE}

Let us consider solar models with fixed chemical composition in the convective envelope (hydrogen and helium abundance $X_{0}, Y_{0}$, respectively). To obtain the solar radius $R$ and luminosity $L$ we vary the hydrogen profile $X(m / M)$ in the core as well as the entropy $s$ of the convective envelope. We use the specific entropy $s$ in the adiabatic part of the convective zone as the parameter of the solar envelope instead of the more conventional mixing-length parameter of convection $\alpha$. The reason is that the value of $\alpha$ depends on the structure of the outermost layers (in particularly on opacity). The specific entropy $s$ is in addition related to the depth of the convection zone depth, but this quantity is also affected by opacity. To compute the specific entropy we use the expression for the free energy of Mihalas et al. (1988), but with omitted electron degeneracy:

$$
s=-\left(\frac{\partial F}{\partial T}\right)_{V, N}=\left(k \sum_{s} n_{s}\left(\frac{5}{2}+\ln \frac{g_{s}}{n_{s} \Lambda_{s}^{3}}\right)+\frac{4}{3} a T^{3}\right) / \rho
$$




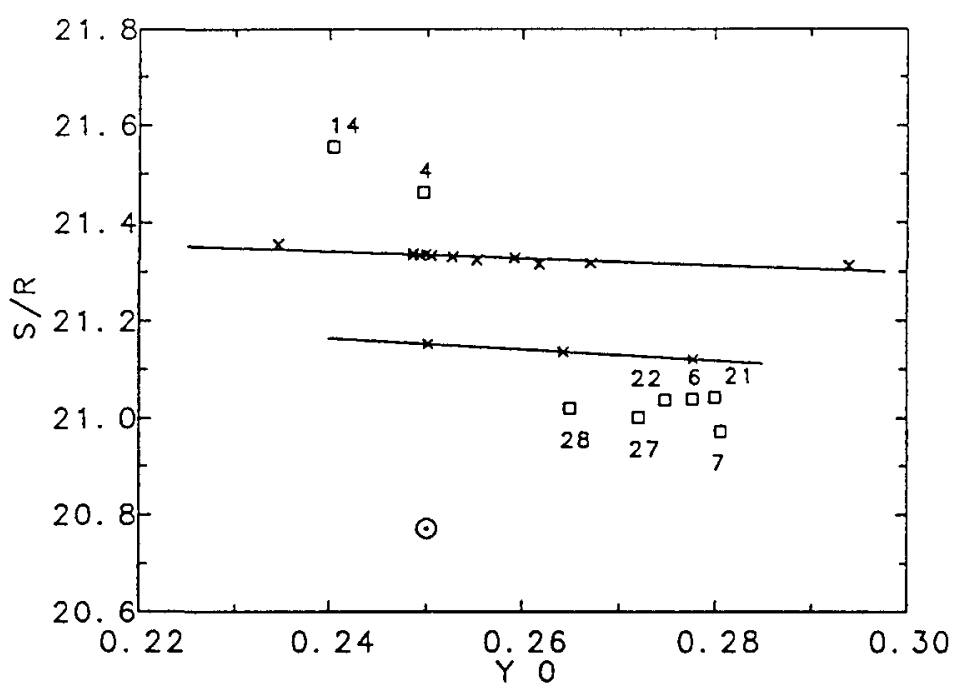

FIGURE I Specific entropy $s / R$ ( $R$ : gas constant) and surface helium abundance. Crosses connected by lines represent the $Y_{0}-s$ relation of our models. Labelled squares denote the models from Christensen-Dalsgaard 1992. $\odot$ represents the helioseismic determination of the envelope parameters (Vorontsov et al. 1991).

where the sum is taken over all particles, $g$, are the partition functions for atoms and ions $\left(g_{0}=2\right.$ for electrons). $\Lambda_{a}$ denotes the thermal wavelength, equal to $h / \sqrt{2 \pi k T m_{s}}$.

We found as a result that the best fit of $s$ turned out to be the same for these models, and all our attempts to construct a solar model with another value of entropy, but constant $Y_{0}$ in the convective envelope, failed. In other words, although there are many models with different $X(m / M)$ profiles in the interior, and the same $Y_{0}$ in the envelope, they have all the same entropy $s$.

\section{RELATION BETWEEN HELIUM AND ENTROPY IN ENVELOPE}

We have also computed solar models with various values of the helium abundance $Y_{0}$ of the envelope. Fitting to solar $R$ and $L$ is again possible, but these models have another entropy $s$ in the convective zone (and consequently another depth of the convective zone $d_{b}$ ). However, for a given helium abundance of the convective zone $Y_{0}$ only one $s$ is allowed, i.e. there is a relation between $Y_{0}$ and the entropy $s$ for a set of models with various $X(m / M)$ profiles in the interior (plotted on Figure I by crosses).

To investigate the sensitivity of this $Y_{0}-s$ relation to the internal physics we have computed models with the opacity altered locally a) in the energygenerating core and $b$ ) in the radiative zone (denoting the region between the energy-generating core and the bottom of the convective zone). If the opacity is changed in the core (where luminosity is not constant), the $Y_{0}-s$ relation is 
preserved. However, changing the opacity in the radiative zone shifts the $Y_{0}-s$ curve as a whole. Thus the opacity in the radiative zone is one of the factors determining the position of the curve on $Y_{0}-s$ plane.

Our calculations were performed with simplified model physics (according to Christensen-Dalsgaard, 1988). The helium abundance and entropy of our evolutionary model do not agree with those obtained in recent evolutionary calculations (Kim et al. 1991, Sackmann et al. 1990, Courtaud et al. 1990, Christensen-Dalsgaard 1991). To reveal the $Y_{0}-s$ relation for other models we have calculated specific entropy for 8 models computed by J. ChristensenDalsgaard (1992). We find a similar relation for three models with different age $(22,6,21)$ and for three models with corrected opacity in the core $(7$, 27,28 ). Model 4 differs from model 6 due to another opacity and model 14 from model 4 because of the inclusion of Coulomb interactions. But all these models have too-high an entropy compared with the value of the helioseismic determination by Vorontsov et al. (1991) (marked by $\odot$ on figure). Preliminary investigations indicate that to reach a low entropy and the required helium content it is necessary to increase opacity in the radiative zone (by a factor of 1.5 or 2) compared with our opacity (based on the simple formulae from Christensen-Dalsgaard 1988). Such an increase would entail a change of opacity and (or) the profile of the hydrogen abundance in the core.

\section{ACKNOWLEDGMENTS}

We thank J.Christensen-Dalsgaard for stimulating discussions and for making his models available to us. We also thank W. Däppen for his help with this poster.

\section{REFERENCES}

Christensen-Dalsgaard, J. 1988, Computational Procedures for GONG Solar Model Project.

Christensen-Dalsgaard, J. 1991, in Challenges to Theories of the Structure of Moderate-mass Stars, pp.11-36, ed. D.O.Gough and J.Toomre (Lecture Notes in Physics, 388, Springer, Heidelberg, 1991).

Christensen-Dalsgaard, J. 1992, Geophys. Astrophys. Fluid Dynamics, in press.

Courtaud, D., Damamme, G., Genot, E., Vuillemin, M., and Turck-Chièze, S. 1990, Solar Phys., 128, 49-60.

Kim, Y.-C., Demarque, P., and Guenther D.B. 1991, $A p J, 377,407-412$.

Mihalas, D., Däppen, W., and Hummer, D.G. 1988, $A p J, 331,815-825$.

Sackmann, I.-J., Boothroyd, A.I., and Fowler W. 1990, ApJ, 360, 727-736.

Vorontsov, S.V., Baturin, V.A., and Pamyatnykh, A.A. 1991, Nature, 349, 4951. 